\title{
Endothelial dysfunction in patients with systemic sclerosis
}

\author{
Renata Pacholczak-Madej ${ }^{1,2}$, Piotr Kuszmiersz ${ }^{3}$, Stanisława Bazan-Socha ${ }^{3}$, Joanna Kosałka-Węgiel ${ }^{3}$, Teresa Iwaniec ${ }^{3}$, \\ Lech Zaręba ${ }^{4}$, Stan Kielczewski ${ }^{1}$, Anna Rams ${ }^{3}$, Jerzy A. Walocha ${ }^{1}$, Jacek Musiał ${ }^{3}$, Jerzy Dropiński ${ }^{3}$
}

${ }^{1}$ Department of Anatomy, Jagiellonian University, Medical College, Krakow, Poland

${ }^{2}$ National Cancer Institute, Maria Skłodowska-Curie Memorial Institute, Krakow Branch, Poland

${ }^{3}$ Department of Internal Medicine, Jagiellonian University, Medical College, Krakow, Poland

${ }^{4}$ Interdisciplinary Centre for Computational Modelling, College of Natural Sciences, University of Rzeszow, Rzeszow, Poland

Adv Dermatol Allergol 2020; XXXVII (4): 495-502

DOI: https://doi.org/10.5114/ada.2019.83501

\begin{abstract}
Introduction: Patients with systemic sclerosis experience endothelial dysfunction and damage even in the absence of clinical manifestations.

Aim: To evaluate various methods for assessing the endothelial function for their applicability to clinical practice. Material and methods: Forty-two patients ( 7 men and 35 women) with systemic sclerosis and 36 controls (11 men and 25 women) matched for age, sex, body mass index, smoking habit, and comorbidities were enrolled in the study. We assessed each participant for typical risk factors for cardiovascular diseases and measured serum levels of vascular cell adhesion molecule-1 (VCAM-1) and thrombomodulin together with flow-mediated dilatation (FMD) of the brachial artery and intima-media thickness (IMT) of the common carotid artery using ultrasonography. Results: Patients with systemic sclerosis did not differ from controls in serum levels of VCAM-1 and thrombomodulin, however, the statistical analysis with adjustment for potential confounders revealed increased levels of thrombomodulin in the patients $(p=0.03)$. They also had a $45 \%$ lower relative increase of FMD (FMD\%), and $13 \%$ higher IMT ( $p<0.01$, both, also after adjustment for potential confounders). In a simple regression model, lower FMD\% was determined by age ( $\beta=-0.57,95 \%$ confidence interval $(\mathrm{Cl}):-0.72$ to -0.43$)$ and $C$-reactive protein levels $(\beta=-0.38,95 \% \mathrm{Cl}:-0.55$ to -0.22$)$. Thicker IMT was related to age $(\beta=0.64,95 \% \mathrm{Cl}: 0.52-0.67)$, glomerular filtration rate $(\beta=-0.34,95 \% \mathrm{Cl}:-0.5$ to -0.18$)$, and blood thrombomodulin levels ( $\beta=0.45,95 \% \mathrm{Cl}$ : $0.13-0.76)$.

Conclusions: Patients with systemic sclerosis present with endothelial dysfunction which may be detected using ultrasonographic methods. The exact mechanism of observed abnormalities is unknown, but it is possibly related to the chronic inflammation and ischemia-reperfusion injury.
\end{abstract}

Key words: atherosclerosis, endothelium, flow-mediated dilatation, intima-media thickness, scleroderma.

\section{Introduction}

Systemic sclerosis (scleroderma) is a chronic disease of the connective tissue characterized by thickening and fibrosis of the skin and internal organs. Historically, there are two major disease subsets: the diffuse type, which is associated with a high risk of pulmonary arterial hypertension, acute renal injury and diffuse skin involvement (extremities proximal to elbows and knees, chest, abdomen and back) [1]; and the limited type, related to sclerodactyly and Raynaud's phenomenon, calcinosis, esophageal dysmotility, telangiectasia and limited skin involvement (face, neck and extremities distal to elbows and knees) [1, 2]. Vascular damage in both systemic sclerosis subsets is mainly microvascular and Raynaud's phenomenon is the earliest manifestation of the disease. The other clinical symptoms related to microvascular abnormalities are digital ulcerations, renal insufficiency and pulmonary arterial hypertension [3, 4]. Macrovascular damage is less frequent but increased stiffness of large arteries, plaque presence and increased intima-media thickness of the common carotid artery (IMT) have also been reported [1, 5, 6]. Systemic sclerosis is associated with an increased risk of myocardial infarct, stroke and peripheral artery disease, although exact mechanisms of these abnormalities remain unknown $[7,8]$.

Address for correspondence: Jerzy Dropiński MD, PhD, Department of Internal Medicine, Jagiellonian University Medical College, 8 Skawińska St, 31-066 Krakow, Poland, phone: +48 1243052 66, e-mail: jerzy.dropinski@uj.edu.pl Received: 22.10.2018, accepted: 14.12.2018. 
Endothelial dysfunction seems to be a key mechanism in the pathophysiology of systemic sclerosis. In many studies the function of the endothelium was measured through flow-mediated dilatation (FMD) of the brachial artery which evaluates the response to shear, the subsequent release of nitric oxide and dilatation of the vessel [9-18]. Several studies have found elevated IMT in patients with systemic sclerosis, which is considered as an indicator of atherosclerosis $[5,9,12,16,18]$. These aforementioned studies, however, conflict in results and were performed on small groups of patients. Endothelial damage can also be assessed through laboratory tests. It is related to the higher expression of adhesion molecules on endothelium, such as E-selectin, endothelin-1, and vascular cell adhesion molecule-1 (VCAM-1) which might be sheared into the blood stream. Activated endothelium also releases other molecules, such as von Willebrand factor, soluble thrombomodulin, and tissue plasminogen factor, which could be the markers of procoagulant activity [19].

\section{Aim}

Taking into account the ambiguous results of previously published studies on endothelial dysfunction in systemic sclerosis, we sought to determine the validity laboratory parameters of endothelium damage, such as serum concentration of thrombomodulin and VCAM-1, as well as to evaluate, in ultrasound, FMD of the brachial artery and measure IMT of the common carotid arteries $[5,9,12,16,18]$. Finally, we analysed their relationships to the clinical features of the systemic sclerosis and antibodies levels. We believe that due to the rarity of that disease every report is valuable.

\section{Material and methods}

Our study follows the case-control observational, retrospective format and received approval from the Bioethics Committee of Jagiellonian University Medical College (9 $9^{\text {th }}$ May 2013, number of protocol: KBET/79/B/2013). All study participants received an explanation of the methodology and safety protocol and gave written consent to be included in the study.

The 42-patient case group was made up of 35 women and 7 men. They were enrolled in the period from 2014 to 2017 at the II Department of Internal Medicine, JagielIonian University, Medical College, Krakow, a diagnostics and treatment centre for autoimmune diseases in southern Poland. These patients were all previously diagnosed with systemic sclerosis according to the criteria of the American College of Rheumatology and all evaluated for the presence of typical systemic sclerosis autoantibodies such as anti-centromere, anti-topoisomerase I (anti-Scl-70, AC-29), anti-RNA polymerase III antibodies, and anti-PM/ Scl antibodies [20]. Candidates for the case group were excluded from the study if they had confirmed atheroscle- rosis, angina pectoris, congestive heart failure, liver failure, uncontrolled hypertension, or cancer. Other comorbidities that did not disqualify a patient but were recorded include hypertension (a history of blood pressure > 140/90 mm Hg or current antihypertensive treatment), hypercholesterolemia (a serum total cholesterol $>5.2 \mathrm{mmol} / /$ or ongoing antihypercholesterolemic therapy), and diabetes mellitus (use of insulin or oral hypoglycaemic agents, or fasting serum glucose $>7.0 \mathrm{mmol} / \mathrm{l}$ ).

The 36-individual healthy control group was made up of 25 women and 11 men that matched the case group as to gender, age, body mass index (BMI) and smoking habit. They were selected with the same exclusion criteria as the studied group and their concomitant morbidities were likewise recorded. The control group was enrolled from the hospital's personnel and their relatives.

\section{Laboratory analysis}

Hospital staff collected fasting blood samples in the morning from the antecubital vein with the least possible tourniquet use. Lipid profile, levels of glucose, alanine transaminase, urine, creatinine for glomerular filtration rate (eGFR), and complete blood count were measured using routine laboratory techniques and C-reactive protein (CRP) with the Johnson \& Johnson VITROS 250. We measured serum parameters specific to this study from blood samples centrifuged in separation tubes at 2,000 $\times \mathrm{g}$ for $10 \mathrm{~min}$ at room temperature within $2 \mathrm{~h}$ of collection. Standardized ELISA method (all, R\&D Systems, Minneapolis, MN, USA) was used in order to measure Interleukin (IL)-6, VCAM-1, and soluble thrombomodulin levels. Antinuclear antibodies (ANA) were analysed using indirect immunofluorescence tests (ThermoFisher, Waltham, USA) and antigen-specific ELISA for autoantibodies against topoisomerase I, centromeres, RNA polymerase III, NOR 90, PM-Scl, Ku, PDGFR, fibrillarin, and Ro-52 (EUROIMMUN, Lübeck, Germany).

\section{Ultrasound examinations}

Two independent experts performed the ultrasound studies using the Siemens Acuson Sequoia 512 with a $10 \mathrm{MHz}$ linear array ultrasonic transducer (Mountain View, Ca, USA). They performed the studies in a dark, quiet room with the subjects in a supine position for $10 \mathrm{~min}$ and having fasted for $10 \mathrm{~h}$. They measured flow-mediated dilation of the brachial artery and intima-media thickness of the carotid artery. They both made three consecutive measurements of every parameter and we recorded the mean of the six measurements for analysis. The ultrasonographers then performed a transthoracic echocardiogram (TTE) on each subject using standard methods [21].

Brachial artery ultrasonography. Celemayer's method was used to measure FMD of the brachial artery [22]. We used the technique described in detail in our previous papers $[23,24]$. The baseline sagittal diameter (D1) of a distal part of the brachial artery was measured in the 
M-presentation with a $10 \mathrm{MHz}$ linear array ultrasonic transducer placed $2-3 \mathrm{~cm}$ proximal to the arterial bifurcation. A sphygmomanometer cuff was then placed on the forearm below the elbow and inflated to $200 \mathrm{~mm} \mathrm{Hg}$ for 5 min and released. One minute after releasing the cuff, the brachial artery diameter was measured a second time (D2) at the same point. FMD is the increase of the brachial artery diameter after deflation of the cuff and is expressed as a percentage of the baseline diameter $(F M D \%=((D 2-D 1) / D 1) \times 100 \%)$. We recorded the mean of the FMD of both arms for analysis.

Intima-media thickness of the common carotid artery. As previously described by us, the IMT of the carotid artery was measured by ultrasound with a $10 \mathrm{MHz}$ linear transducer. The thickness of the anterior and posterior walls of the common carotid arteries was measured in the longitudinal projection immediately proximal to their bifurcation $[23,24]$. We recorded the mean value of the IMT on the right and left side for analysis.

\section{Statistical analysis}

The results of the case and control groups were compared using Statistica 12.5 software. The continuous variables all had non-normal distribution according to the Shapiro-Wilk test. We report them here as median and interquartile ranges and compare them using the MannWhitney U-test. Categorical variables are given as percentages and compared by $\chi^{2}$ test. Age, sex, BMI, and comorbidities, such as arterial hypertension, diabetes mellitus, and hypercholesterolemia were considered as potential confounders. Therefore, the main outcome results: FMD\%, IMT, VCAM-1, and thrombomodulin were log-transformed and a one way covariance analysis (ANCOVA) was performed to adjust for them. The univariate linear regression models with adjustment for BMI, age, and sex were used to analyse associations between two selected parameters. Markers of endothelial injury were assessed using simple regression models. Results were considered significant when the $p$-value was less than 0.05 .

\section{Results}

\section{Characteristics of patients and controls}

Table 1 presents characteristics of patients with systemic sclerosis and controls. As it has been shown, both groups were similar in age, sex, BMI, smoking habits and comorbidities (hypertension, diabetes mellitus and hypercholesterolemia).

Characteristics of the patients with systemic sclerosis at the time of evaluation are given in Table 2. The median duration of the disease was 4 (range: $1-7$ ) years and the majority of the patients $(69 \%, n=29)$ had a diffuse type of the disease. All of the systemic sclerosis patients had positive results of antinuclear antibodies in indirect immunofluorescence. The disease specific anti-topoisom- erase I antibodies were present in half of them. Other types of antibodies, such as anti-centromere, anti-RNA polymerase III, anti-Ro-52, and anti-Ku antibodies had lower prevalence. Raynaud's phenomenon was the most common clinical manifestation, followed by interstitial lung disease, skin lesions on the fingers, and pulmonary arterial hypertension. Half of the patients with systemic sclerosis were being treated with systemic glucocorticosteroids at the time of evaluation. They were also receiving or had received other immunosuppressive agents such as azathioprine, cyclophosphamide, methotrexate, mycophenolate mofetil, or rituximab. Among other medications, the most common were statins, $\beta$-blockers, angiotensin converting enzyme inhibitors or angiotensin receptor antagonists, diuretics and calcium channel blockers.

\section{Basic laboratory tests and basic transthoracic echocardiographic parameters}

Table 1 presents the results of basic laboratory tests and TTE parameters. As expected, patients with systemic sclerosis had higher inflammatory markers, such as CRP and IL-6, as well as a lower haemoglobin level. Surprisingly, individuals in the control group were characterized by a higher total cholesterol level and creatinine concentration (in all subjects in normal range).

In TTE, patients with systemic sclerosis had a slightly lower ejection fraction, thicker interventricular septum and higher systolic pulmonary artery pressure (Table 1). Moreover, those with the diffuse type of the disease were characterized by larger left atrium (3.9 (3.5-4.2) vs. 3.9 (3.8-3.9) $\mathrm{cm}, p=0.005)$ and left ventricle (both, diastolic diameter $4.6(4.4-4.9)$ vs. $4.3(4.2-4.6) \mathrm{cm}, p=0.04$ and systolic diameter $3(2.9-3.1)$ vs. $2.9(2.8-2.9) \mathrm{cm}, p=0.02)$, thicker interventricular septum (1.1 (1-1.2) vs. $0.9(0.8-1.1) \mathrm{cm}$, $p=0.003)$ and thicker left ventricle posterior wall (1 (0.9-1.1) vs. $0.8(0.9-0.9) \mathrm{cm}, p=0.007)$.

\section{Laboratory markers of endothelial injury}

Patients with systemic sclerosis had similar serum levels of VCAM-1 and thrombomodulin as compared to healthy individuals (Table 3). However, the ANCOVA analysis performed with adjustment for potential confounders revealed that levels of thrombomodulin, but not VCAM-1, were increased in the systemic sclerosis group $(p=0.03)$. Both laboratory markers of endothelial injury remained in a strong positive association between each other in patients and in controls ( $\beta=0.58,95 \%$ confidence interval $(\mathrm{Cl})$ : 0.4-0.76, $\beta=0.44,95 \% \mathrm{Cl}$ : 0.28-0.6, respectively).

In systemic sclerosis patients, we documented a strong positive association between thrombomodulin and kidney function $(\beta=0.84,95 \% \mathrm{Cl}$ : 0.53-1.15, $\beta=0.69,95 \% \mathrm{Cl}: 0.44-0.93$, for creatinine and urea blood levels, respectively). For VCAM-1 such relation was 
Table 1. A summary of demographic, laboratory and echocardiographic parameters in patients with systemic sclerosis and controls

\begin{tabular}{|c|c|c|c|}
\hline Parameter & $\begin{array}{l}\text { Patients } \\
(n=42)\end{array}$ & $\begin{array}{l}\text { Controls } \\
(n=36)\end{array}$ & $P$-value \\
\hline Age [years] & $63.5(49-70)$ & $57(47-65)$ & 0.3 \\
\hline Male gender, $n(\%)$ & $7(16.7)$ & $11(30.6)$ & 0.15 \\
\hline Body mass index [kg/m²] & $25(23.3-27.5)$ & $26.9(23.7-28.7)$ & 0.2 \\
\hline Hypertension, $n$ (\%) & $17(40.5)$ & $10(27.8)$ & 0.18 \\
\hline Diabetes mellitus, $n$ (\%) & $5(11.9)$ & $4(11.1)$ & 0.87 \\
\hline Hypercholesterolemia, $n$ (\%) & $15(35.7)$ & $8(22.2)$ & 0.18 \\
\hline Smoking currently, n (\%) & $2(4.8)$ & $4(11.1)$ & 0.68 \\
\hline Smoking in the past, $n(\%)$ & $12(28.6)$ & $11(30.6)$ & 0.89 \\
\hline Smoking [packs/years] & $0(0-5)$ & $0(0-3.5)$ & 0.8 \\
\hline \multicolumn{4}{|l|}{ Basic laboratory tests: } \\
\hline High-density lipoprotein [mmol/l] & $1.3(1.1-1.5)$ & $1.4(1.2-1.8)$ & 0.12 \\
\hline Triglycerides [mmol/l] & $1.3(1.1-2.1)$ & $1.2(0.8-1.8)$ & 0.16 \\
\hline Glucose [mmol/l] & $4.8(4.4-5)$ & $5(4.8-5.2)$ & 0.11 \\
\hline Creatinine $[\mathrm{mmol} / \mathrm{l}]$ & $67(58-80)$ & $74(67-88)$ & $0.03^{*}$ \\
\hline Urea [mmol/l] & $5.2(4.3-6.1)$ & $4.9(4.2-5.5)$ & 0.32 \\
\hline Alanine transaminase [U/I] & $23.5(18.5-33)$ & $23.5(14.9-30)$ & 0.48 \\
\hline C-reactive protein [mg/dl] & $6.8(5-18.7)$ & $1.3(1-2.6)$ & $<0.01^{*}$ \\
\hline Interleukin-6 [pg/ml] & $3.6(2.5-10)$ & $1.8(1.3-2.3)$ & $<0.01^{*}$ \\
\hline \multicolumn{4}{|l|}{ Echocardiographic parameters: } \\
\hline Left ventricular diastolic diameter [cm] & $4.6-(4.3-4.9)$ & $4.7(4.5-4.9)$ & 0.13 \\
\hline Left ventricular systolic diameter [cm] & $3(2.9-3.1)$ & $3(2.9-3.1)$ & 0.56 \\
\hline Right ventricular diameter $[\mathrm{cm}]$ & $2.1(1.9-2.3)$ & $2.1(1.9-2.3)$ & 1 \\
\hline Left atrial diameter [cm] & $3.7(3.3-4)$ & $3.7(3.6-4)$ & 0.76 \\
\hline Left ventricle posterior wall thickness [cm] & $1(0.9-1.1)$ & $0.9(0.8-1)$ & 0.14 \\
\hline Interventricular septal thickness [cm] & $1(0.9-1.2)$ & $0.9(0.9-1.1)$ & $0.04^{*}$ \\
\hline Ejection fraction (\%) & $65(60-67)$ & $68(67-68)$ & $<0.01^{*}$ \\
\hline Systolic pulmonary artery pressure [mm Hg] & $36(32-42)$ & $32(29-34)$ & $<0.01^{*}$ \\
\hline
\end{tabular}

Categorical variables are presented as numbers (percentages), continuous variables as median and interquartile range. The results which are statistically significant are marked *. $n$-number.

demonstrated only for serum creatinine concentrations ( $\beta=0.67,95 \% \mathrm{Cl}: 0.39-0.95)$. Additionally, thrombomodulin in the patients with systemic sclerosis was related to the interventricular septum and posterior wall thickness $(\beta=0.78,95 \% \mathrm{Cl}: 0.5-1.06, \beta=0.83,95 \% \mathrm{Cl}: 0.56-1.1$, respectively), as well as the CRP level $(\beta=0.57,95 \% \mathrm{Cl}$ : 0.31-0.83)

Patients with systemic or limited disease had similar VCAM-1 and thrombomodulin levels. Likewise, comorbidities, treatment mode and type of antibodies had no impact on either laboratory marker of endothelial damage.

\section{Ultrasound parameters of endothelial injury}

As shown in Table 3, patients with systemic sclerosis were characterized by $45 \%$ lower FMD\% and $13 \%$ higher IMT, as compared to the control group ( $p<0.01$, both, also after adjustment for potential confounders). Moreover, both these unfavourably changed ultrasound parameters of endothelial injury remained in a mutual relationship to each other, but only in the patient group ( $\beta=-0.48,95 \% \mathrm{Cl}:-0.63$ to -0.32 ). In a simple regression model lower FMD\% was determined by age $(\beta=-0.57,95 \% \mathrm{Cl}:-0.72$ to -0.43$)$ and CRP $(\beta=-0.38$, $95 \% \mathrm{Cl}:-0.55$ to -0.22$)$. Thicker IMT was also related to age ( $\beta=0.64,95 \% \mathrm{Cl}: 0.52-0.67)$, to impaired kidney function (for eGFR, $\beta=-0.34,95 \% \mathrm{Cl}$ : -0.5 to -0.18 ), and to thrombomodulin blood levels $(\beta=0.45,95 \% \mathrm{Cl}$ : 0.13-0.76).

For TTE parameters, IMT was positively associated with interventricular septum thickness $(\beta=0.28$, 
95\% Cl: 0.07-0.49), and posterior wall thickness $(\beta=0.24,95 \% \mathrm{Cl}: 0.03-0.45)$ in systemic sclerosis, while for FMD\% such associations were not documented. IMT, but not FMD\% was also related to the thrombomodulin level ( $\beta=0.39,95 \% \mathrm{Cl}$ : 0.11-0.67) and duration of glucocorticosteroids treatment $(\beta=0.31,95 \% \mathrm{Cl}$ : 0.1-0.52) in the patient group.

Patients with systemic or limited systemic sclerosis did not differ in FMD\% or IMT. Similarly, disease duration, treatment mode, or type of antibodies had no impact on these variables.

Finally, none of the analysed comorbidities had any influence on FMD\%, but IMT was greater in those with hypertension (0.06 (0.05-0.065) vs. 0.075 (0.065-0.085), $p=0.004)$.

\section{Discussion}

In this study we have shown that patients with systemic sclerosis present with endothelial dysfunction which is demonstrated by decreased FMD\% and increased IMT compared to well-matched controls. Also, the thrombomodulin level, a laboratory marker of endothelium damage, was higher in the patient group, after adjustment for potential confounders. As expected, parameters of endothelial dysfunction were determined by age, but also by variables related to the specificity of the disease, such as kidney injury and inflammatory markers. Another interesting finding of this study is that IMT is associated with the interventricular septal and posterior wall thicknesses, and that it correlates with the thrombomodulin level. This relationship has not been reported before and allows us to speculate that endothelium damage might be followed by a remodelling of the large vessels wall, leading to increased stiffness of the arteries and higher afterload, as well as hypertrophy of the heart structures. Parameters evaluated in our study were related to the inflammatory markers, but did not depend on systemic sclerosis clinical manifestation, type of immunosuppressive treatment used currently or in the past, and type of antibodies present in the subgroups analysis.

Many previous reports have shown decreased FMD in patients with systemic sclerosis [5, 9, 12-14]. In some studies, however, the results were contradictory $[15,16]$. This discrepancy might be explained by the patient selection. In studies with negative results, more subjects presented limited systemic sclerosis, suggesting that the diffuse type is more prone to endothelial dysfunction. Our study contradicts this hypothesis because parameters of endothelial injury were comparable in subjects with a limited and diffuse subtype of disease, although the number of enrolled subjects with the limited form was much smaller than those with diffuse disease.

Increased IMT is a diagnostic tool which indicates ongoing, subclinical atherosclerosis. Our results are consistent with previous reports documenting thicker IMT in systemic
Table 2. Clinical characteristics of the patients with systemic sclerosis $(n=42)$

\begin{tabular}{|c|c|}
\hline Parameter & Patients \\
\hline Duration of the disease [years] & $4(1-7)$ \\
\hline Limited disease & $13(30.9)$ \\
\hline Diffuse disease & $29(69.1)$ \\
\hline Antinuclear antibodies presence, $n(\%)$ & $42(100)$ \\
\hline Anti-topoisomerase I antibodies presence, $n(\%)$ & $18(42.9)$ \\
\hline Anti-PM/Scl antibodies presence, $n(\%)$ & $5(11.9)$ \\
\hline Anti-centromere antibodies presence, $n(\%)$ & $9(21.4)$ \\
\hline RNA polymerase III antibodies presence, $n(\%)$ & $1(2.4)$ \\
\hline Anti-Ku antibodies presence, $n(\%)$ & $3(7.1)$ \\
\hline \multicolumn{2}{|l|}{ Organ involvement: } \\
\hline Digital ulcers, $n(\%)$ & $12(28.6)$ \\
\hline Abnormal nailfold capillaries, $n$ (\%) & $9(21.4)$ \\
\hline Fingertip lesions, $n(\%)$ & $17(40.5)$ \\
\hline Telangiectasia, $n(\%)$ & $6(14.3)$ \\
\hline Raynaud's phenomenon, $n(\%)$ & $33(78.6)$ \\
\hline Dysphagia, $n(\%)$ & $13(31)$ \\
\hline Interstitial lung disease, $n(\%)$ & $25(59.5)$ \\
\hline \multicolumn{2}{|l|}{ Treatment characteristic: } \\
\hline Current glucocorticosteroids, $n(\%)$ & $20(47.6)$ \\
\hline $\begin{array}{l}\text { Current glucocorticosteroids dose, recalculated } \\
\text { to methylprednisolone }[\mathrm{mg} / \mathrm{day}]\end{array}$ & $2(0-4)$ \\
\hline Systemic glucocorticosteroids therapy [years] & $1(0-3)$ \\
\hline \multicolumn{2}{|c|}{ Immunosuppressive treatment (currently or in the past): } \\
\hline Azathioprine, $n$ (\%) & $3(7.1)$ \\
\hline Cyclophosphamide, $n(\%)$ & $48(42.9)$ \\
\hline Methotrexate, $n(\%)$ & $6(14.3)$ \\
\hline Mycophenolate mofetil, $n(\%)$ & $8(19)$ \\
\hline Rituximab, $n$ (\%) & $15(35.7)$ \\
\hline \multicolumn{2}{|l|}{ Internal medicine medications: } \\
\hline $\begin{array}{l}\text { Angiotensin converting enzyme inhibitors or } \\
\text { angiotensin receptor antagonists, } n(\%)\end{array}$ & $15(35.7)$ \\
\hline Statins, $n(\%)$ & $11(26.2)$ \\
\hline$\beta$-Blockers, $n(\%)$ & $9(22.4)$ \\
\hline Diuretics, $n(\%)$ & $11(26.2)$ \\
\hline Calcium channel blockers, $n(\%)$ & $15(35.7)$ \\
\hline
\end{tabular}

Categorical variables are presented as numbers (percentages), continuous variables as median and interquartile range. $n$-number.

sclerosis $[9,11]$. However, Szucs et al. and Domsic et al. demonstrated that IMT was similar in patients with systemic sclerosis and controls [11, 18]. Andersen et al. combined ultrasonographic and laboratory tests in order to evaluate endothelial function and have found the elevated level of soluble E-selectin and VCAM-1 in patients with systemic sclerosis with an inverse correlation to the FMD\% [15]. 
Table 3. Parameters of endothelial injury in patients with systemic sclerosis and controls

\begin{tabular}{|c|c|c|c|}
\hline Variable & $\begin{array}{l}\text { Patients } \\
(n=42)\end{array}$ & $\begin{array}{l}\text { Controls } \\
(n=36)\end{array}$ & $P$-value \\
\hline \multicolumn{4}{|l|}{ Laboratory parameters of endothelial injury: } \\
\hline Vascular cell adhesion molecule-1 [ng/ml] & $834.6(703.2-1037.3)$ & $817.7(714.2-921.8)$ & 0.36 \\
\hline Thrombomodulin [ng/ml] & $4.8(3.9-5.5)$ & $4.4(3.9-4.9)$ & 0.31 \\
\hline \multicolumn{4}{|l|}{ Ultrasound parameters of endothelial injury and atherosclerosis: } \\
\hline Relative increase of flow mediated dilatation of a brachial artery & $5.5(2.6-8.3)$ & $10(8.5-11.4)$ & $<0.01^{*}$ \\
\hline Median value of intima-media thickness of a common carotid artery $[\mathrm{cm}]$ & $0.075(0.06-0.08)$ & $0.065(0.06-0.085)$ & 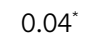 \\
\hline
\end{tabular}

The aetiology of endothelial damage and atherosclerosis in systemic sclerosis might be related to multiple factors, including inflammation, traditional cardiovascular risk factors and the influence of ischemia with subsequent reperfusion. In our study, both groups were similar in terms of cardiovascular risk factors (BMI, gender, smoking habits, comorbidities). Individuals in the control group had slightly higher total cholesterol and lowdensity lipoprotein levels. This may be due to a higher prevalence of statins intake by patients with systemic sclerosis because the prevalence of hypercholesterolemia was similar in both groups. Higher creatinine levels in the controls (but within normal limits) are possibly due to greater muscle mass, although both groups had similar BMI. Traditional cardiovascular risk factors do not alone explain the accelerated atherosclerosis seen in patients with systemic sclerosis. The prevalence of hypertension, obesity, hypercholesterolemia, and diabetes were similar in other cohort studies [7, 25]. In fact, cardiovascular risk factors were less prevalent in patients with systemic sclerosis in the Australian Systemic Sclerosis Cohort Study, suggesting that other factors are responsible for the observed abnormalities [8]. Mok et al. hypothesized that systemic sclerosis is an independent determinant of coronary calcification as it is observed in the skin of affected subjects [25].

Inflammation is well established as a factor leading to vascular dysfunction and atherosclerosis. Premature atherosclerosis has been observed in patients with chronic inflammatory diseases such as systemic lupus erythematosus, antiphospholipid syndrome or systemic vasculitides [26-30]. We also documented higher CRP and IL-6 levels in the case group. Therefore, we may postulate that chronic inflammation accelerates atherosclerosis in systemic sclerosis.

Chronic endothelial damage is also caused by ischemia and reperfusion which lead to dysfunction and loss of cell integrity and tissue injury. In systemic sclerosis, tissue hypoxia together with chronic blood flow reduction related to microvascular abnormalities is a stimulus for the increased expression of vascular endothelial growth factors (VEGF) and angiogenesis. VEGF also contributes to the development of fibrosis [31, 32].

Timar et al. found that rosuvastatin improves FMD, corrects unfavourable changes in the blood lipid profile, and decreases CRP, but does not reduce carotid atherosclerosis after a 6-month treatment period in patients with systemic sclerosis [33]. In their study, Kuwana et al. conclude that statins may be beneficial in treating vascular manifestations such as Raynaud's phenomenon [34]. Therefore, statins should be advised in most patients with systemic sclerosis for endothelial protection but more clinical trials are needed to verify this hypothesis [35]. The other options in the treatment of endothelial dysfunction in systemic sclerosis are phosphodiesterase type 5 enzyme inhibitors (sildenafil, tadalafil), synthetic analogues of prostacyclin, and bosentan - an endothelin receptor antagonist. They lead to the smooth muscle relaxation with subsequent vasodilatation, and might be considered in the treatment of severe Raynaud's phenomenon, digital ulcers and pulmonary arterial hypertension [36-38]. The patients enrolled in our study did not use the abovementioned medications, but their impact on parameters of endothelial dysfunction is an interesting subject for further research. Obviously, patients benefit most from optimal immunosuppressive treatment that controls inflammation and prevents consequences of clinically active systemic sclerosis.

\section{Conclusions}

In this study we have demonstrated that patients with systemic sclerosis present with endothelial dysfunction which may be detected using ultrasonographic methods. FMD and IMT are easy tools with diagnostic and therapeutic relevance, which can help elicit a group of subjects with subclinical atherosclerosis. Measurements of selected adhesion molecule blood levels might give conflicting results and should only be used in research. The exact mechanism of the observed disturbances is unknown, however, we may speculate that it is due to chronic inflammation and ischemia-reperfusion 
injury. Although large observational studies are needed to verify this hypothesis, it seems that proper immunosuppressive treatment might influence endothelium injury indirectly by reducing inflammatory response in systemic sclerosis subjects.

\section{Acknowledgments}

This project was funded by the Jagiellonian University Medical College, No. K/ZDS/007897 (to R.P.). The funder had no role in the study design, data collection and analysis, decision to publish, or preparation of the manuscript.

\section{Conflict of interest}

The authors declare no conflict of interest.

\section{References}

1. Nussinovitch $U$, Shoenfeld Y. Autoimmunity reviews atherosclerosis and macrovascular involvement in systemic sclerosis: myth or reality. Autoimmun Rev 2011; 10: 259-66.

2. Lambova S. Cardiac manifestations in systemic sclerosis. World J Cardiol 2014; 6: 993-1005.

3. McGoon MD, Kane GC. Pulmonary hypertension: diagnosis and management. Mayo Clinic Proceedings 2009; 84: 191207.

4. Ngian G, Sahhar J, Wicks IP, et al. Cardiovascular disease in systemic sclerosis - an emerging association? Arthritis Res Ther 2011; 13: 237.

5. Timár O, Soltész P, Szamosi S, et al. Increased arterial stiffness as the marker of vascular involvement in systemic sclerosis. J Rheumatol 2008; 35: 1329-33.

6. Zeng Y, Li M, Xu D, et al. Macrovascular involvement in systemic sclerosis : evidence of correlation with disease activity. Clin Exp Rheumatol 2012; 30: 76-80.

7. Man A, Zhu Y, Zhang Y, et al. The risk of cardiovascular disease in systemic sclerosis: a population-based cohort study. Ann Rheum Dis 2013; 72: 1188-93.

8. Ngian GS, Sahhar J, Proudman SM, et al. Prevalence of coronary heart disease and cardiovascular risk factors in a national cross-sectional cohort study of systemic sclerosis. Ann Rheum Dis 2012; 71: 1980-3.

9. Lekakis J, Mavrikakis M, Papamichael C, et al. Short-term estrogen administration improves abnormal endothelial function in women with systemic sclerosis and Raynaud's phenomenon. Am Heart J 1998; 136: 905-12.

10. Lekakis J, Papamichael C, Mavrikakis M, et al. Effect of longterm estrogen therapy on brachial arterial endothelium-dependent vasodilation in women with Raynaud's phenomenon secondary to systemic sclerosis. Am J Cardiol 1998; 9149: 1555-7.

11. Szucs G, Tımar O, Szekanecz Z, et al. Endothelial dysfunction precedes atherosclerosis in systemic sclerosis - relevance for prevention of vascular complications. Rheumatology 2007; 46: 759-62.

12. Bartoli F, Blagojevic J, Bacci M, et al. Flow-mediated vasodilation and carotid intima-media thickness. Ann N Y Acad Sci 2007; 290: 283-90.
13. D’Andrea A, Caso P, Cuomo S, et al. Myocardial and vascular dysfunction in systemic sclerosis: the potential role of noninvasive assessment in asymptomatic patients. Int J Cardiol 2007; 18: 298-301.

14. Cypiene A, Laucevicius A, Venalis A, et al. The impact of systemic sclerosis on arterial wall stiffness parameters and endothelial function. Clin Rheumatol 2008; 27: 1517-22.

15. Andersen GN, Mincheva-nilsson L, Kazzam E, et al. Assessment of vascular function in systemic sclerosis indications of the development of nitrate tolerance as a result of enhanced endothelial nitric oxide production. Arthritis Rheum 2002; 46: 1324-32.

16. Roustit M, Simmons G, Baguet J, et al. Discrepancy between simultaneous digital skin microvascular and brachial artery macrovascular post-occlusive hyperemia in systemic sclerosis. J Rheumatol 2008; 35: 1576-83.

17. France Y, Rossi P, Granel B, et al. Endothelial function and hemodynamics in systemic sclerosis. Clin Physiol Funct Imaging 2010; 30: 453-9.

18. Domsic R, Dezfulian C, Shoushtari A, et al. Endothelial dysfunction is present only in the microvasculature and microcirculation of early diffuse systemic sclerosis patients. Clin Exp Rheumatol 2014; 32: 154-60.

19. Deanfield JE, Halcox JP, Rabelink TJ. Endothelial function and dysfunction: testing and clinical relevance. Circulation 2007; 115: 1285-95.

20. Salehi-abari I. 2017 ACR/EMA Revised criteria for too early diagnosis of Granulomatosis with Polyangiitis (GPA) 2017 ACR/EMA Revised Criteria for too Early Diagnosis of Granulomatosis with Polyangiitis (GPA). Autoimmune Dis Ther Approaches 2016; 3: 127.

21. Hillis GS, Bloomfield P. Basic transthoracic echocardiography. BMJ 2005; 330: 1432-6.

22. Healy B, Ojrio C. Endothelial cell dysfunction: an emerging endocrinopathy linked to coronary disease. JACC 1990; 16: 7-8.

23. Pacholczak R, Bazan-Socha S, Iwaniec T, et al. Endothelial dysfunction in patients with granulomatosis with polyangiitis: a case-control study. Rheumatol Int 2018; 38: 1521-30.

24. Pacholczak R, Bazan-Socha S, Iwaniec T, et al. Endothelial dysfunction in patients with eosinophilic granulomatosis with polyangiitis. Clin Rheumatol 2019; 38: 417-24.

25. Mok MY, Lau CS, Chiu SSH, et al. Systemic sclerosis is an independent risk factor for increased coronary artery calcium deposition. Arthritis Rheum 2011; 63: 1387-95.

26. Wang DG, Tang XW, Fan Y, et al. Decreased flow-mediated dilatation in patients with systemic lupus erythematosus: a meta-analysis. Inflammation 2014; 37: 2067-75.

27. Ahmadi B, Bonakdar ZS, Hashemi SM, et al. Endothelial dysfunction in Iranian lupus patients. Rheumatol Int 2011; 31: 27-31.

28. Cugno M, Borghi MO, Lonati LM, et al. Patients with antiphospholipid syndrome display endothelial perturbation. J Autoimmun 2010; 34: 105-10.

29. Talc M, Poredo P, Peternel P, et al. Endothelial function is impaired in patients with primary antiphospholipid syndrome. Thromb Res 2006; 118: 455-61.

30. Cohen Tervaert JW. Cardiovascular disease due to accelerated atherosclerosis in systemic vasculitides. Best Pract Res Clin Rheumatol 2013; 27: 33-44.

31. Cannarile F, Valentini V, Mirabelli G, et al. Cardiovascular disease in systemic sclerosis. Ann Transl Med 2015; 3: 8. 
32. Farouk HM, Hamza SH, El Bakry SA, et al. Dysregulation of angiogenic homeostasis in systemic sclerosis. Int J Rheum Dis 2013; 16: 448-54.

33. Timár O, Szekanecz Z, Kerekes G, et al. Rosuvastatin improves impaired endothelial function, loSwers high sensitivity CRP, complement and immuncomplex production in patients with systemic sclerosis - a prospective case-series study. Arthritis Res Ther 2013; 15: R105.

34. Kuwana M, Okazaki Y, Kaburaki J. Long-term beneficial effects of statins on vascular manifestations in patients with systemic sclerosis. Arthritis Rheum 2009; 60: 448.

35. Kowal-Bielecka O, Bielecki M, Kowal K. Recent advances in the treatment of systemic sclerosis. Pol Arch Med Wewn 2013; 123: 51-8.

36. Kowal-bielecka O, Fransen J, Avouac J, et al. Update of EULAR recommendations for the treatment of systemic sclerosis. Ann Rheum Dis 2017; 76: 1327-39.

37. Teloken PE, Mulhall JP. Impact of phosphodiesterase type 5 inhibitors on endothelial function. Rev Urol 2008; 10: 26-30.

38. Oskolkova O, Sarich N, Tian Y, et al. Incorporation of iloprost in phospholipase-resistant phospholipid scaffold enhances its barrier protective effects on pulmonary endothelium. Sci Rep 2018; 8: 879. 